\title{
The Recognition Method of Radiation Source Based on Information Entropy and Cloud Model
}

\author{
Yun $\mathrm{Lin}^{1,2,3}$, Can Wang ${ }^{2}$, Chunguang $\mathrm{Ma}^{1 *}$, Zheng $\mathrm{Dou}^{2}$, Zhiqiang $\mathrm{Wu}^{2}$, \\ Zhiping Zhang ${ }^{2}$ \\ ${ }^{1}$ College of Computer Science and Technology, Harbin Engineering University, \\ Harbin, China \\ ${ }^{2}$ College of Information and Communication Engineering, Harbin Engineering \\ University, Harbin, China \\ ${ }^{3}$ Department of Electrical Engineering, Wright State University, Dayton, Ohio, \\ U.S. \\ douzheng@hrbeu.edu.cn, Corresponding Author: machunguang@hrbeu.edu.cn, \\ zhiqiang.wu@wright.edu
}

\begin{abstract}
Information entropy features are often used for radiation source signal recognition, but due to the information entropy is very sensitive to noise, so this method has greater recognition rate changes with the SNR. This paper putting forward a viable recognition based on Entropy and cloud model. using cloud model to extract secondary features of signals, build radiation source signal's entropy and cloud feature vector. The method uses cloud model description and processing interval fuzzy and observation noise data, better solve the low SNR cases of radiation source signal feature extraction problem. At the same time, putting forward the similar cloud classification recognition algorithm based on cloud model. The simulation results show that Entropy and cloud model has better recognition effect under low SNR, which can improve the signals' recognition rate under low SNR.
\end{abstract}

Keywords: Information entropy; cloud model ; Radiation source recognition; Similar cloud

\section{Introduction}

In general, electronic receiver receive signals over a wide frequency range, and therefore it may receive many different types of signal. To monitor, analyze, interfere or demodulation these signals, we must accurately identify the modulation type of the signal. Given any period of the received signal, we can judge the type of the signal when the information unknown or incomplete, and then extract the appropriate modulation parameters to provide a reference for the next signal demodulation. Therefore, it has been a important studies to the radiation signal recognition on electronic warfare. Early as the nineteen sixties, C.S.Waver had published a report on pattern recognition to achieve modulation signal recognition [1-5]. Since then, the theoretical study on modulation recognition developed rapid, and research method is also increasing[6].

With the development of information theory, it has more and more studies in signal feature extraction. Information entropy can measure the signal's degree of uncertainty and complexity, it is a characteristic index of signal complexity, Therefore, the information can be used to describe the signal"s contains information, so it provides a theoretical

\footnotetext{
${ }^{1}$ Chunguang $\mathrm{Ma}$ is the corresponding author.
} 
basis for signal characterization description. The literature[7] using fuzzy neural networks method to recognize the radar emitter, this method is simple, convenient, fast and accurate, especially for complex recognition. The literature[8] provide pattern recognition method and parameter estimation methods for AM, MASK, MFSK, MPSK. With the development of information theory, entropy analysis method has been widely used in various fields: The literature[9] introduce the information entropy development process, including the development process, concepts, characteristics and application methods; The literature[10] use the entropy to extract the signal's internal characteristic also achieved good results; The literature [11] introduce the concept of entropy to determine the optimal grouping histogram for radar signal feature extraction and analysis; The literature [12] study on entropy feature which is applied to evaluate of machine rotating vibration; The literature[13] proposed by using the fuzzy entropy to extract the rotor rubimpact voice signal's characteristic parameters and the method to identify different rub impact condition. For using the different characteristics to the signal feature extraction. The literature[14] describes the application of wavelet entropy in feature extraction; The literature[15] using approximate entropy (ApEn) and norm entropy (NoEn) to form the feature vector and applied to recognize radar emitter signals; The literature[16] using entropy analysis method applied to the feature extraction of the radiation source, when the SNR is greater than $10 \mathrm{~dB}$, the signal can be well distinguish by entropy curve, but when SNR is low recognition rate dropped sharply, cannot effectively distinguish signals, but the recognition rate will decline sharply under low SNR environment.

This paper aiming at using entropy feature for radar emitter signal recognition get low recognition rate under low SNR, putting forward a new method to extract cloud feature of information entropy by using cloud model, and then using the similar cloud classifier to distinguish signals. Through the Matlab simulation, the results show that the new method can greatly improve the recognition rate of radar emitter signal under low SNR rate.

\section{Cloud Model}

\subsection{The Basic Concept of Cloud Model}

Cloud model[17-20] is a description of qualitative concept and quantitative concept uncertain transforming model which proposed by professor Li Deyi, it's used to describe the fuzziness and randomness as well as the relationship between two concept. Cloud Model use three digital characteristics parameters expectation Ex, entropy En and hyper entropy $\mathrm{He}$ to the overall characterization of cloud. The normal cloud model is the most commonly used.

The definition of cloud model: If $\mathbf{U}$ is a numeric representation of the quantitative domain, $\mathbf{C}$ is a qualitative concept in $\mathbf{U}$, If the quantitative value $x \in \mathbf{U}$ is a stochastic implementation of qualitative concept, $x$ is membership of $\mathbf{C}, \mu(x) \in[0,1]$ is random number with stable tendency,

$$
\begin{aligned}
& \mu: \mathbf{U} \rightarrow[0,1] \\
& \forall x \in \mathbf{U}, x \rightarrow \mu(x)
\end{aligned}
$$

The distribution of $x$ is called cloud in the field $\mathrm{U}$ and denoted as $\mathbf{C}(\mathbf{X})$. Each one is called a cloud droplet.

\subsection{Digital Characteristics of the Cloud Model}

Based on the normal distribution function and normal membership function, cloud model with three digital characteristics parameters expectation $E x$, entropy En and hyper entropy $\mathrm{He}$ to express a qualitative concept and reflect the uncertainty of the concept. 
Expectation Ex reflect the focus of the cloud droplet, it is able to represent the qualitative concept points in the domain space, and it is the most typical sample point concept of quantization.

Entropy En reflect the qualitative concept's uncertainty degree, It represent the value range which can be accepted by the qualitative concept in the domain space, that is the fuzzy degree.

Hyper entropy $\mathrm{He}$ is a measure of the entropy's uncertainty, it is the randomness of cloud droplet samples, and it reflects the degree of the cloud droplet's discrete. It is related to the randomness and fuzziness.

\subsection{Digital Characteristics of the Cloud Model}

The cloud model can be achieved by cloud generator. Cloud generator divided into FCG(Forward Cloud Generator) and BCG(Backward Cloud Generator).

The FCG is shown in Figure 1, according to the digital characteristic's distribution of known cloud droplet group, and then generate the cloud droplet which meet distribution characteristic.

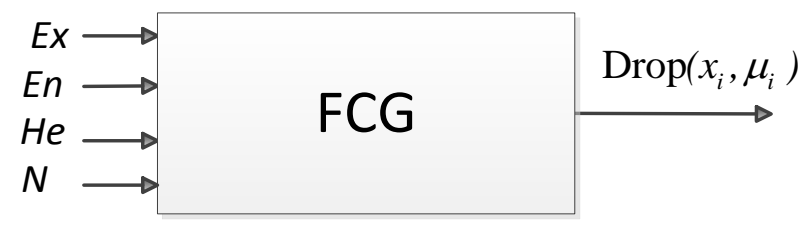

\section{Figure 1. Forward Cloud Generator}

Input: Three digital characteristics and the cloud droplet number $\mathrm{N}$.

Output: the cloud droplet's quantitative value $\operatorname{Drop}\left(x_{i}, \mu_{i}\right), i=1,2, \cdots, N$.

In general, the one-dimensional normal cloud generator needs to follow the normal distribution of the " $3 E n "$ principle, in the cloud droplet group, the probability distribution within " $[E x-3 E n, E x+3 E n]$ " is $99.7 \%$, so outside the " $3 E n$ " cloud droplets are small probability events, usually it can be ignored. The FCG specific calculation process is shown as follows:

1. Taking $E n$ as expectation, $H e$ for standard deviation to generate normal random number $y_{i}$.

2. Taking $E x$ as expectation, $y_{i}$ for standard deviation to generate normal random number $x_{i}$.

3. According to step 1 and step 2, computing the membership $x_{i}$ to $\mu_{i}$.

$\mu_{i}=\exp \left[-\frac{\left(x_{i}-E x\right)^{2}}{\left(2 y_{i}\right)^{2}}\right]$

The BCG is shown in Figure 2, which according to the known numerical of cloud droplet, it can calculate cloud droplet's distribution characteristics and digital characteristics, it is a transformation process from quantitative to qualitative. 


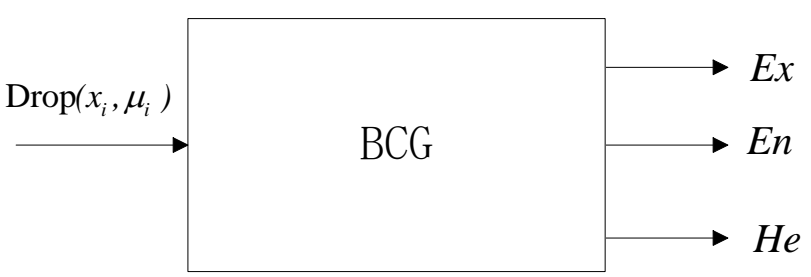

Figure 2. Backward Cloud Generator

Input: Cloud droplets and cloud droplets' membership degree $\mu_{i}, i=1,2, \cdots, N$.

Output: Cloud's digital characteristics $(\mathrm{Ex}, \mathrm{En}, \mathrm{He})$.

If we know $\mathrm{N}$ cloud droplets' membership information, then specific calculation process of BCG is shown as follows:

1、Calculating $\mathrm{N}$ cloud droplets' expectation Ex.

2、From formula 1 , it can get the intermediate variables.

$$
y_{i}=\frac{\left|x_{i}-E x\right|}{\sqrt{-2 \ln \mu_{i}}}
$$

3、 Calculating the entropy of cloud droplets.

$$
E n=\frac{\sum_{i=1}^{N} y_{i}}{N}
$$

4、 Calculating the hyper entropy of cloud droplets.

$$
H e=\sqrt{\frac{\sum_{i=1}^{N}\left(y_{i}-E n\right)^{2}}{N-1}}
$$

\subsection{Similar Cloud}

From the concept of cloud model, qualitative different concepts can be expressed with different cloud, but for the same kind of qualitative concept can be formed by a plurality of cloud to express, how to examine the association between these cloud, it involves the similarity of these clouds, how to measure the degree of similarity between the cloud. Because every cloud are available three digital characterize to express its overall characteristics, therefore we can study the similarity problem between different cloud according to the characteristic parameter of the cloud. If more than one cloud describe a qualitative concept, there exists similarity between these cloud. Here the "similarity" refers to the similarity threshold is smaller than a given between clouds. For two different parameters cloud, if the similarity between them is less than a given threshold, it can be considered that the two cloud is similar cloud or equivalent cloud.

In theory, the cloud droplet number is infinite which we used to describe a qualitative concept, but we could not produce an infinite number of cloud droplets, it can only be approximated by the finite cloud droplets, this leads to the two cloud digital characterizes can not be exactly the same, just similar. The more the number of cloud droplets, the two cloud similarity is more close to the theoretical value, when the similarity is less than a threshold value, we can think it is equivalent to two cloud. Therefore, the similarity degree not only depends on the characteristics of cloud, but also the cloud droplet number. The literature [21] presents a similar cloud algorithm based on distance; The literature [22] given another similar cloud algorithm which based on cloud droplet membership; The [23] put forward the similarity algorithm based on expectation curve and the similarity algorithm based on maximum boundary; In order to reduce the running 
time of the program calculation and program, this paper simplifies the similar cloud algorithm to literature [21].

Similar cloud algorithm:

1、Input two cloud digital characteristic $E x_{1}, E n_{1}, H e_{1}, E x_{2}, E n_{2}, H e_{2}$ and appropriate threshold $\delta$.

2、According to the digital characteristics produce cloud droplet the number of $\mathrm{N}$.

$$
\begin{aligned}
\operatorname{Drop}\left(x_{1}, \mu_{1}\right) & =\operatorname{FCG}\left(E x_{1}, E n_{1}, H e_{1}, N\right) \\
\operatorname{Drop}\left(x_{2}, \mu_{2}\right) & =\operatorname{FCG}\left(E x_{2}, E n_{2}, H e_{2}, N\right)
\end{aligned}
$$

3、Ascending order of the cloud $\operatorname{Drop}\left(x_{1}, \mu_{1}\right), \operatorname{Drop}\left(x_{2}, \mu_{2}\right)$.

4、 Calculating the distance between two clouds

$$
D=\sqrt{\left(\sum_{i=1}^{N}\left[x_{1}(i)-x_{2}(i)\right]^{2}\right)}
$$

5、According to similarity threshold to determine the similarity degree between two clouds.

\section{The second Characteristic Extraction based on Cloud-Model}

At present, the cloud model theory has been widely applied in many fields. The fuzziness (the bigger the numerical ranges, the fuzzy the concept)and randomness (random membership of the object to describe) of the object to describe are fully integrated together, and constitute a mapping between qualitative (the concepts of the domain) and quantitative (on the attribute value in the domain of membership), so it provides a good theoretical basis for the feature extraction of radiation source. The literature[24] introduced the concept of cloud model in artificial immune system, using multiple recognition to distinguish the uncertain antigen combined with cloud recognition and achieved good results; The [25] joined the cloud model algorithm in emitter recognition, cloud model is used to deal with the random and fuzzy problems caused by noise.

\subsection{Information Entropy of Radiation Source}

Firstly, the paper extract the entropy feature of nine kinds of common radiation source, including AM, FM, PM, 2ASK, 2FSK, 2PSK, QPSK, step frequency signal (SF) and linear frequency modulation signal (LFM), then used the Shannon entropy $H_{1}$ and exponent entropy $H_{2}$ to constitute a group of two-dimensional feature vector, the simulation results show that, the two-dimensional entropy characteristics of nine kinds of radiation source signal has good cohesive collection sex and better separability in the high SNR. 


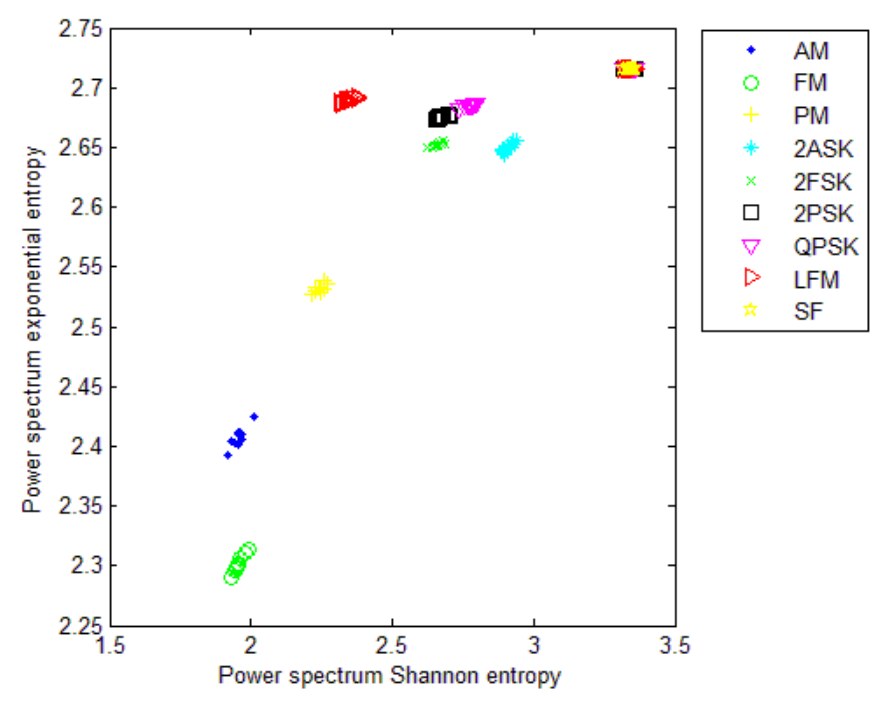

Figure 3. Two Dimensional Characteristics of Entropy(SNR $=5 \mathrm{~dB})$

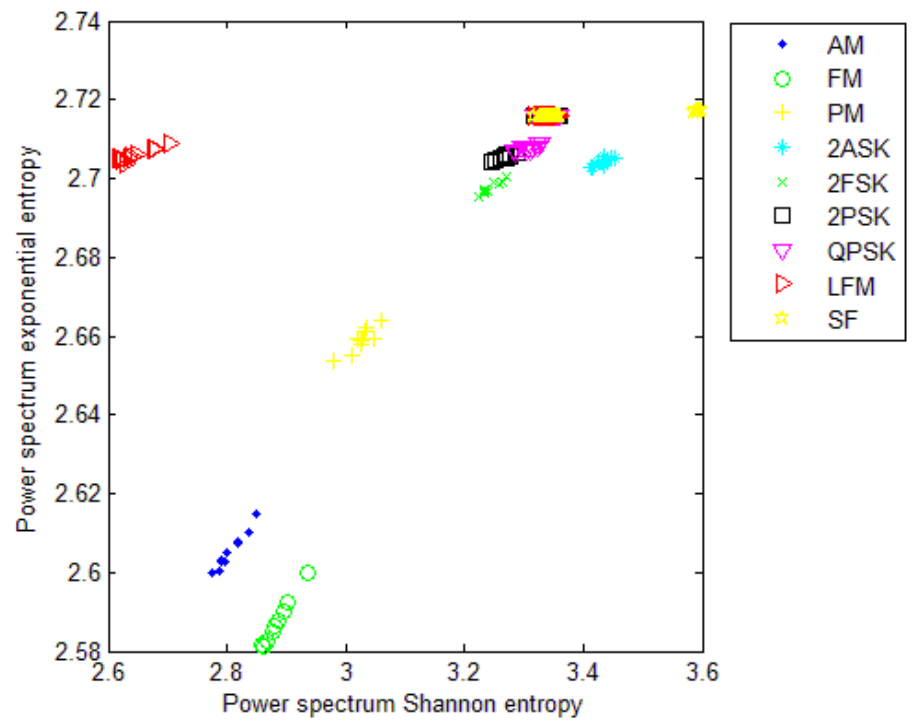

Figure 4. Two Dimensional Characteristics of Entropy(SNR=0dB) 


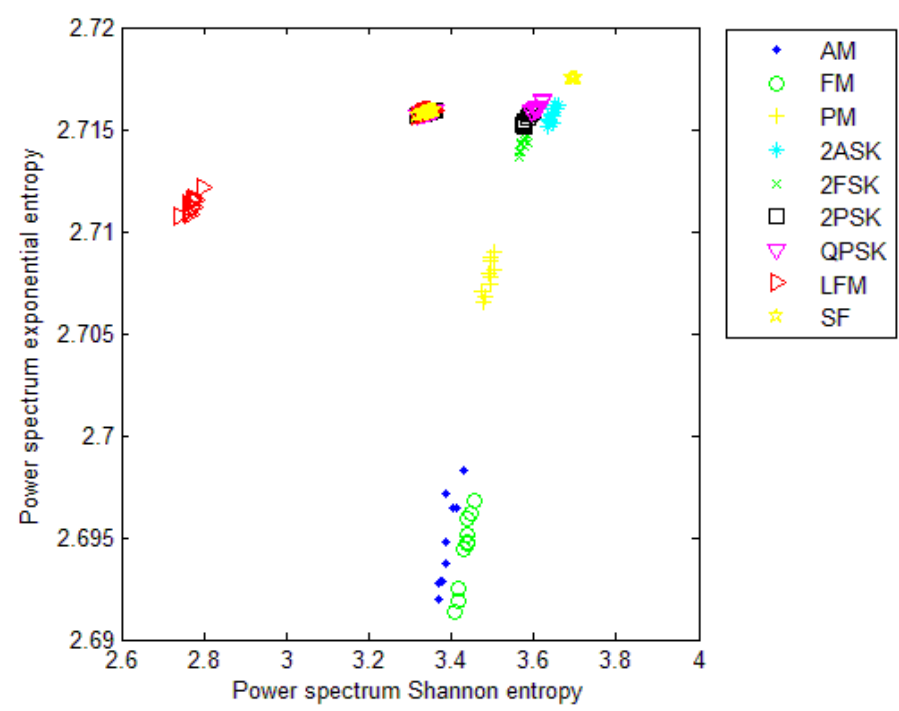

Figure 5. Two Dimensional Characteristics of Entropy (SNR=-5dB)

From Figure 3, 4 and 5 we can see that the separability of characteristics of twodimensional entropy is better in $5 \mathrm{~dB}$ SNR, but when the SNR dropped to $0 \mathrm{~dB}$, or $-5 \mathrm{~dB}$, although the concentration of entropy is well, when the entropy of different signal added together, the inter class separation degree decreased sharply.

\subsection{Construction of the Cloud Model}

It can be seen from the definition of the normal cloud model theory, for the distribution of the normal cloud model, cloud droplets are in accord with normal distribution. If the signal characteristic parameters change with the variation of SNR, the characteristic value fluctuate near to a stable value, and the distribution of accord with normal distribution, So, it can use the digital characteristic parameters of Ex, En and $H e$ to reflect the entropy's changes of unstable, thus to extract fine characteristic parameters of different radiation sources signals.

The membership degree is a very important point to cloud model construction. From the distribution of the signal's entropy, the Shannon entropy and the index entropy are approximately obey the normal distribution, We can use an entropy which through the normalized as the membership degree function. Two dimensional entropy characteristic vector $\left[H_{1}, H_{2}\right]$ form cloud droplet $\operatorname{Drop}\left(x_{i}, \mu_{i}\right)$. Finally, using the BCG to extract the digital characteristics.

\subsection{Classification Algorithm based on Similar Cloud}

From signal's entropy characteristic extraction to Entropy-Cloud model transformation, entropy change, and then to the template matching of Similar cloud computing, and final to the classification, the whole system model as shown in Figure 6. 


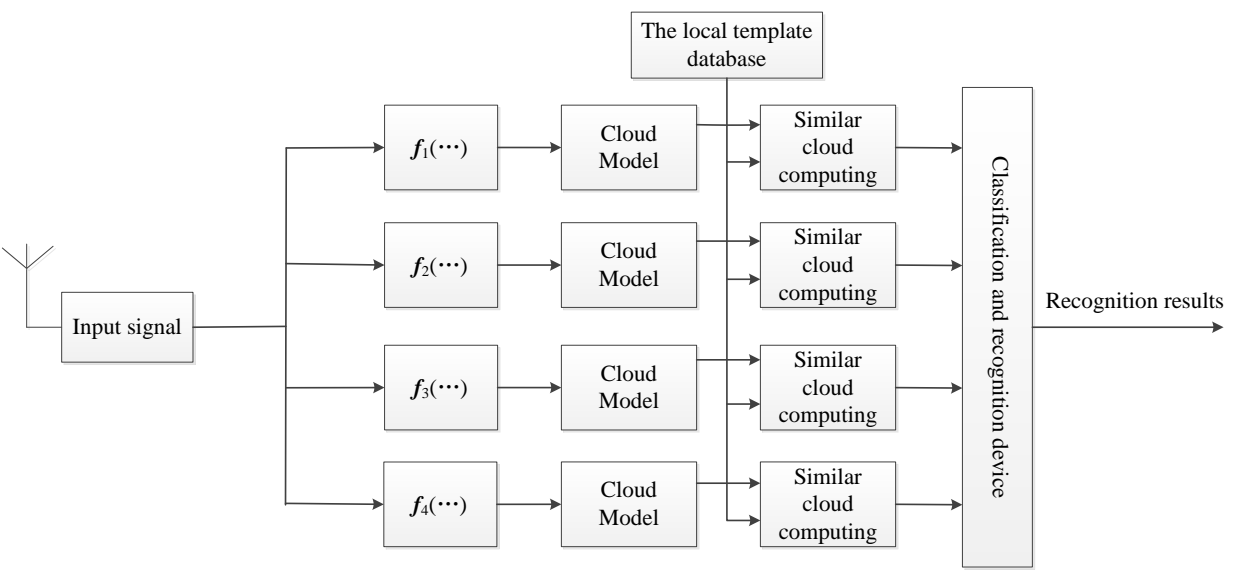

Figure 6. System Model Figure

In Figure 6 , the $f_{1}(\cdots), f_{2}(\cdots), f_{3}(\cdots) 、 f_{4}(\cdots)$ are power spectrum Shannon entropy, power spectrum exponential entropy, singular spectrum Shannon entropy, singular spectrum exponential entropy.

Similar cloud matching and data classification algorithm:

1. Setting up a signal match with the template $L$ times, we can get

$\mathbf{D}=\left[\begin{array}{cccc}d_{11} & d_{12} & \cdots & d_{1 K} \\ d_{21} & d_{22} & \cdots & d_{2 K} \\ \vdots & \vdots & \vdots & \vdots \\ d_{L 1} & d_{L 2} & \cdots & d_{L K}\end{array}\right]$

$K$ represents the number of local signal in the matrix.

2、 Making a recognition calculation to the template matrix $\mathbf{D}$

$\mathbf{S}(i, j)=\sqrt{\frac{\min D(i)-D(i, j)}{\min D(i)}}$

We select an appropriate threshold, if S satisfies

$\mathbf{S}(i, j)=\sqrt{\frac{\min D(i)-D(i, j)}{\min D(i)}} \leq \delta$

We think this is the match signal, and set to 1 , otherwise it set to 0 .

3. Counting the results of $L$ times similar cloud computing, then calculating the number of each signal's match times

$$
\begin{gathered}
\mathbf{T}=\mathbf{S}^{\prime} ; \\
\mathrm{P}(n)=\sum_{i=1}^{L} T(i) / L
\end{gathered}
$$

We can get

$$
\mathbf{P}=\left[P_{1}, P_{2}, \cdots P_{K}\right]
$$

\section{Simulation Results}

Using 9 kinds of radiation source signals of AM, FM, PM, 2ASK, 2FSK, 2PSK, QPSK, LFM, step frequency signal as an example. Setting the threshold value is 0.05 , nine signals recognition rate curve as shown in Figure 8. 


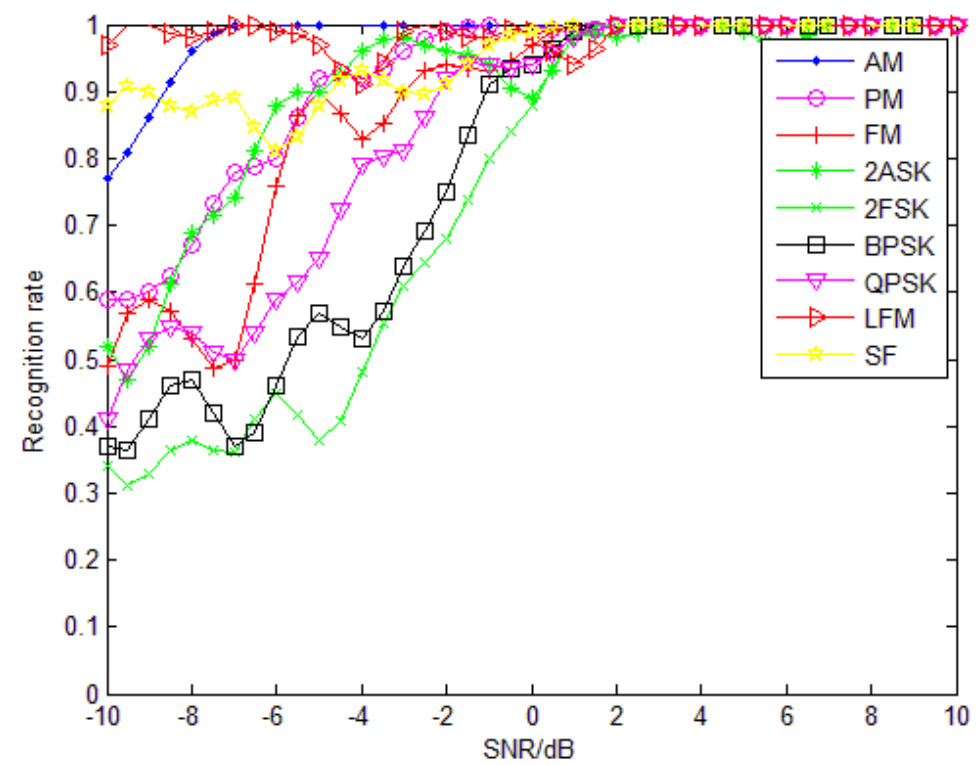

Figure 7. Nine Signals Recognition of Entropy and Cloud system

In the process of simulation, each signal simulat100 times at each SNR. It can be seen from Figure 7, nine signal recognition rate can reach $95 \%$ in $0 \mathrm{~dB}$, of which AM, PM, FM, 2ASK, LFM, step frequency signal the recognition rate can reach $90 \%$ in the $-5 \mathrm{~dB}$. It shows that the recognition method of Entropy-Cloud model can improve signal effectively in low SNR.

\section{Simulation Results}

This paper introduce the cloud model and put forward a kind of algorithm to extract the second characteristics based on entropy. This method firstly extracted entropy characteristics of the signal, and then transform the entropy characteristics of cloud model, finally using the similar cloud and classification to classify and identify the signal. Through the simulation results we can draw the conclusion, the method of cloud model can effectively deal with the entropy values of random and fuzzy problems in low SNR, and the Entropy-Cloud model radiating source recognition system can effectively identify the signal.

\section{Acknowledgment}

This work was supported by the Key Development Program of Basic Research of China(JCKY2013604B001), the Nation Nature Science Foundation of China (No.61301095 and No.61201237), Nature Science Foundation of Heilongjiang Province of China (No. QC2012C069 and F201408) and the Fundamental Research Funds for the Central Universities (No. HEUCF1508).

Meantime, all the authors declare that there is no conflict of interests regarding the publication of this article.

\section{References}

[1] S. Z Hsue. S.S Soliman. "Automatic modulation classification using zero crossing". IEEE Proceedings on Radar and Signal Processing, , vol. 137, no. 6, (1990), p. 460.

[2] E.E Azzouz , A.K Nandi, "Procedure for automatic recognition of analogue and digital modulations", IEEE Proceedings on Communications, , vol. 143, no. 5, (1996), p. 260. 
[3] A.K Nandi, E.E Azzouz E.E, "Algorithms for automatic modulation recognition of communication signals[J]. IEEE Transactions on Communications", vol. 46, no. 4, (1998), p. 431.

[4] Y. Han, G. Wei, C. Song, et al. "Hierarchical digital modulation recognition based on higher-order cumulants[C]", 2012 Second International Conference on Instrumentation, Measurement, Computer, Communication and Control (IMCCC), (2012), p. 1645.

[5] B. Lifeng, Y. Ning. "Automatic Modulation Identification for Communication System[J]", Radio communication technology, vol. 37, no. 4, (2011), pp. 59.

[6] S. Yong-qian, Xi Liang. "Real-valued Immune Detector Dimension-reducing Optimization Algorithm with Entropy[J]", Journal of Harbin University of Science and Technology , (2014),02:pp. 68-72.

[7] W. Jian-hua,Z. Li-ping,Y. Ping-liang,S. Yan-ji, "A method for Radar Emitter Recognition Based on Fuzzy Neural Network", (1999), 02:67-69+73.

[8] L. Na, "Study of Modulation Identification and Signals Feature Extraction”.Xi'an: Xi'an Electronic and Science University.(2008).

[9] Z. Jiguo, V. P.Singh. "The theory and application of information entropy[M]", Beijing: Water Conservancy and electric Chinese press,(2012):31.

[10] L. Shizhong, J. Xiaojun, Z. Sulei, "The Application Study of Entropy Analysis Method in Feature Extraction[J]", Journal of North China Institute of Technology, vol. 20, no. 3, (1999), pp. 279-281.

[11] Y. Fei, L. J-qing, "Radar signal parameter feature extraction algorithm based onentropy optimal classification[J]"., Modern defence technology, vol. 36, no. 1, (2008), pp. 98-99.

[12] S. Tao, H. Shuhong, H. Shoumu, Y. Shuzi, "Extracting Information Enyropy Features for Rotating Machiner Vibration Signals[J]”, Chinese Journal of Mechanical Engineering, vol. 37, no. 6, (2001), p. 94.

[13] D. Aili, Z. Li, B. Yongqiang, "Recognition of Rub-impact Acoustic Emission Signal Based on Fuzzy Entropy[J]", Journal of Mechanical Engineering", vol. 46, no. 3, (2010), p. 71.

[14] L. Jianxun, K. Xizhen, G. Hua, "The Application of Wavelet Variance and Wavelet Entropy in Signal Feature Extraction[J]", Journal of Xi'an University of Technology, vol. 23 no. 4, (2007), pp. 365.

[15] Z. Ge-xiang,H. Lai-zhao, J. Wei-dong. "Radar emitter signal recognition based on entropy features[J]"., Chinese Journal of Radio Science, vol. 20, no. 4, (2005), pp. 441-444.

[16] L. Jingchao, L. Yibing, L. Yun, "The application of Entropy Analysis in Radiation Source Feature Extraction[J]", Journal of Projectiles, Rockets,Missiles and Guidance, vol. 31, no. 5, (2011), pp. 156157.

[17] Y. Qiong, L. Shaowen, Z. Youhua, "Cloud model and application overview[J]”, Computer Engineering and Design, vol. 32, no. 12, (2011), p. 4198.

[18] L. Deyi, L. Changyu, "The universality of the normal cloud model theory", Engineering Science, vol. 6 , no. 8, (2004), p. 31.

[19] L. Deyi, M. Haijun, S. Xuemei, "Cloud model and cloud model generator Cloud model and cloud model generator[J]", Computer R\&D, vol. 32, no. 6, (1995), pp. 15-18.

[20] L. Deyi, D. Yi, "The uncertainty of artificial intelligence", BeiJing:National Defense Industry Press.(2005).

[21] Z. Yong, Z. Dong ning, L. De yi, "The Similar Cloud and the Measurement Method[J]”, Information and Control, vol. 33, no. 2, (2004), p. 130.

[22] H. Hai-sheng,W. Ru-chuan, "Subjective trust evaluation model based on membership cloud theory[J]", Journal on Communications, vol. 29, no. 4, (2008), pp. 14-18.

[23] L. Hai-lin,G. Chong-hui,Q. Wang-ren, "Similarity Measurement between Normal Cloud Models[J]", Acta Electronice Sinica, vol. 39, no. 11, (2011), pp. 2562-2565.

[24] W. Si Peng, "Detecting Technology Based on Cloud Model[J]", Computer Technology and Development, vol. 16, no. 6, (2006), pp. 188-190.

[25] L. Hai-jun, L. Zheng, J.Wen-li, "A Method for Emitter Recognition Based on Cloud Model[J]”, Journal of Electronics \& Information Technology, vol. 31, no. 9, (2009), pp. 2798-2803. 